\title{
El conocimiento procedimental en jugadores de voleibol de categoría infantil
}

\section{Procedural knowledge in under-14 category volleyball players}

\author{
Claver, F. ${ }^{1}$, Fernández-Echeverría, C. ${ }^{1}$, Gil, A. ${ }^{2}$, Moreno, A ., Moreno, M.P . \\ ${ }^{1}$ Universidad de Extremadura; ${ }^{2}$ Universidad Católica San Antonio de Murcia \\ Fecha de envio: 2/12/2013; Aceptado: 20/05/2015
}

\begin{abstract}
Resumen: El propósito fundamental de este estudio fue analizar el conocimiento procedimental (CP) de los 306 jugadores de voleibol (M: 13.61; DT: .796), participantes en el Campeonato de Espańa de Selecciones Autonómicas de categoría infantil del año 2011, en función de la experiencia federada y el rendimiento en clasificación. La variable dependiente fue el conocimiento procedimental ("saber cómo"). Para su medida se empleó el cuestionario de conocimiento procedimental (CCPV; Moreno, Del Villar, García-González, García-Calvo, \& Moreno, 2013). Las variables independientes fueron la experiencia en práctica federada, medida a través del número de años de en competición federada y el rendimiento, entendido como el puesto que ocupó cada equipo en la clasificación final del campeonato. Los resultados mostraron la existencia de diferencias significativas en el conocimiento procedimental, en función de la experiencia federada y del rendimiento. Además, el conocimiento, la experiencia federada y el rendimiento se asociaron de forma significativa. Estos resultados se discuten con estudios previos que ponen de manifiesto la relevancia de las variables conocimiento procedimental, experiencia y rendimiento en el proceso que lleva a los jugadores hacia la excelencia deportiva. Se considera necesario que los entrenadores tengan en cuenta estas tres variables de estudio a la
\end{abstract}

hora de proponer tareas de entrenamiento que sirvan para optimizar en el proceso de iniciación deportiva.

Palabras clave: conocimiento procedimental, experiencia, rendimiento, voleibol.

Abstract: The main aim of this study was to analyze the procedural knowledge (PK) of the 306 players (M: 13.61; SD: .796), from de Spanish Championship in Under-14 category depending on de federated experience and performance. The dependent variable was procedural knowledge ("know how") measured trough the Procedural Knowledge Questionnaire (Alberto Moreno et al., 2013). The independent variables were experience in federated practice and performance. Results showed statistical differences in procedural knowledge due to experience and performance. In addition, were also found the relationships between procedural knowledge, experience and performance. These results agree with previous studies that highlighted the importance of these variables into the road to the sport excellence. It is necessary for trainers to take these variables in order to propose specifically training tasks to optimize players' formative process. Keywords: procedural knowledge, experience, performance, volleyball.

\section{Introducción}

Los deportes colectivos, como el voleibol, están compuestos principalmente por habilidades abiertas, en las que el individuo debe presentar constantemente soluciones adaptativas a las alternativas que se le presentan en juego. En este sentido, la perspectiva ecológica ha tratado de explicar el proceso de adquisición de pericia en el deporte sin el procesamiento de la información, asumiendo que los sujetos pueden percibir directamente las propiedades significativas del ambiente sin procesos mediadores ni interpretación de información. De este modo, cuando el sujeto se desenvuelve en el ambiente recibe información suficiente para captar e interpretar los estímulos sin necesidad de la intervención de representaciones mentales (Araújo, 2006; Araujo \& Davids, 2009). Uno de los conceptos más importantes que desarrolla esta teoría son las affordances (posibilidades de acción) que representan las posibilidades de acción dentro de un contexto dado, y estas posibilidades de acción se perciben directamente en función del significado que los datos ambientales tienen para el deportista, obteniendo las propiedades del entorno como re-

Dirección para correspondencia [Correspodence address]: Fernando Claver Rabaz. Avda. Universidad s/n. Facultad de Ciencias del Deporte. Uex. (Espańa). E-mail: fclaver@unex.es sultado de medidas funcionales y significativas para el sujeto (Araújo, 2005; Araújo, Davids, y Hristovski, 2006). En este sentido, los deportistas expertos son aquellos con un comportamiento visual más global, donde la categorización es un fenómeno básico, siendo la pericia perceptiva un proceso de acoplamiento entre la percepción y la categorización (Araújo, Davids, Cordovil, Ribeiro \& Fernandes, 2009).

Desde una perspectiva ecológica, el conocimiento significa el grado de adecuación del ejecutante con su entorno. Se distingue entre conocimiento perceptivo o "conocimiento del" entorno y conocimiento simbólico o "conocimiento sobre" el entorno. Esta distinción deja entrever la que tanto el conocimiento sobre cómo actuar (conocimiento de) y conocimiento sobre como verbalizar las representaciones memorísticas (conocimiento sobre) se encuentran dentro del proceso de percepción-acción (Araújo et al., 2009). La bibliografía relativa al paradigma ecológico destaca que el conocimiento de un deportista puede mejorar sus habilidades, ya que ayuda al jugador a leer patrones de juego, predecir movimientos de los jugadores o percibir información sobre su oponente (Araujo \& Davids, 2009).

De forma paralela, la psicología cognitiva, ha destacado la influencia del conocimiento y la toma de decisiones del de- 
portista en la pericia deportiva (para una revisión, Williams \& Ericsson, 2005). Los investigadores señalan que los deportistas requieren una atención selectiva elevada que permita la captación y el procesamiento de los estímulos más relevantes de la situación de juego, con el propósito de tomar una decisión óptima en el menor tiempo posible (Tenenbaum, Yuval, Elbaz, Bar-Eli, \& Weinberg, 1993). Pero, en el deporte, no solo importa velocidad de la toma de decisiones, sino que el deportista, tras seleccionar la mejor opción que se adapte al contexto, deberá ejecutarla (García-López, González-Víllora, Gutiérrez, \& Serra, 2013). Igualmente, el conocimiento del deportista sobre su deporte influye en sus procesos cognitivos, de tal modo que dirige la atención, el comportamiento visual, el reconocimiento, la anticipación y por último, la selección de la respuesta y su ejecución. De forma inversa, el proceso de selección de la respuesta y su ejecución refinan los procesos intermedios y también la representación de problemas del deportista (MacMahon \& McPherson, 2009). Así, la base de conocimiento a la que accede un deportista y el uso de estrategias y táctica depende en gran medida del contexto de juego, que está condicionado por el entorno, el deportista y la tarea (Newell, 1996). Como consecuencia, los deportistas usarán procesos de actualización sobre las tendencias del oponente, mediante la visualización de su comportamiento en situaciones reales de juego (McPherson \& MacMahon, 2008).

Dentro del paradigma del conocimiento, los investigadores han conceptualizado diferentes tipos de conocimiento: declarativo (Anderson, 1987; Chi, 1978), procedimental (Abernethy, Thomas, \& Thomas, 1993; Anderson, 1987), estratégico(Chi, 1978), metacognitivo (Abernethy et al., 1993; Browne, Carlson, \& Hastie, 2004) y condicional (Alexander \& Judy, 1988). La teoría de Control Adaptativo del Pensamiento Racional (ACT-R: Anderson, Knowles, \& Gilbourne, 2004) una de las más aceptadas dentro de la psicología cognitiva, hace énfasis en dos tipologías de conocimiento fundamentales: conocimiento declarativo, identificado con el "saber qué" (Anderson, 1987; McPherson, 1994; Thomas \& Thomas, 1994) y conocimiento procedimental, identificado con el "saber cómo" (Abernethy et al., 1993; Thomas, 1994). El conocimiento procedimental ha sido el más referenciado, puesto que es entendido como el conocimiento sobre cómo actuar y hacer algo (Abernethy, Farrow, \& Berry, 2003). En voleibol, el conocimiento procedimental está representado por procedimientos de actuación, tales como ubicarse en una zona determinada del campo para defender un ataque del contrario ante un bloqueo mal formado o enviar el balón de saque hacia determinadas zonas del campo contario que se encuentran menos cubiertas.

Los investigadores han analizado la relación existente entre conocimiento procedimental, experiencia y rendimiento, destacando el conocimiento como uno de los elementos más importante que caracterizan a los deportistas expertos, y a aquellos deportistas que más rinden considerándose que éstos poseen un conocimiento procedimental superior, más organizado, estructurado y jerarquizado (McPherson, 1999; Moreno, Moreno, García-González, García-Calvo, \& Del Villar, 2010; Thomas, French, Thomas, \& Gallagher, 1988).

En cuanto a la relación del conocimiento con la experiencia se ha puesto de manifiesto la importancia de la experiencia en práctica federada, medida a partir de los años de práctica y de las horas acumuladas en entrenamiento, como variables fundamentales en el desarrollo del conocimiento procedimental en el deporte (Gil et al., 2013; Iglesias, García-González, García-Calvo, León, \& Del Villar, 2010; MacMahon \& Starkes, 2008). De este modo, diversas han sido las investigaciones, que los deportistas con más experiencia poseen un mayor nivel de conocimiento (Del Villar, Iglesias, Moreno, Fuentes, \& Cervelló, 2004; Macquet, 2009; McPherson \& MacMahon, 2008). En voleibol, en categorías de formación, otros estudios han obtenido diferencias significativas en el conocimiento en función de los años de experiencia en práctica federada (Fernández-Echeverría, Moreno, Gil, Claver, \& Moreno, 2014; Gil, Moreno, Moreno, García-González, \& Del Villar, 2011a; Moreno, Moreno, Iglesias, García-González, \& Del Villar, 2006).

Otros estudios se han centrado en analizar la relación entre el rendimiento en clasificación y el conocimiento (Moreno, Moreno, García-González, Iglesias, \& Del Villar, 2006). Los resultados obtenidos en dicho trabajo determinaron la existencia de una relación lineal y significativa entre las variables conocimiento procedimental y rendimiento, de manera que a mayor conocimiento, mejor fue la posición que ocuparon en la clasificación final.

El propósito fundamental de este estudio fue analizar el conocimiento procedimental de jugadores de voleibol, en función de su experiencia en práctica federada y su rendimiento en competición. A partir de este objetivo general se plantearon las siguientes hipótesis:

- Existirán diferencias en el conocimiento procedimental de los jugadores en función de la experiencia en práctica federada, mostrando un conocimiento procedimental superior los jugadores con mayor experiencia en práctica federada.

- Existirán diferencias en el conocimiento procedimental de los jugadores en función del rendimiento en clasificación, mostrando un conocimiento procedimental superior los jugadores pertenecientes a equipos mejor clasificados.

- Existirá asociación significativa entre el conocimiento procedimental, la experiencia en práctica federada y rendimiento en clasificación. 


\section{Método}

\section{Participantes}

La muestra de estudio estuvo compuesta por un total de 306 jugadores de voleibol de categoría infantil (M: 13.61; DT: .796), pertenecientes a los 32 equipos (14 masculinos y $18 \mathrm{fe}$ meninos) participantes en el Campeonato de España de Selecciones Autonómicas de 2011, celebrado en Cartagena (España). En el estudio participaron jugadores con una experiencia en práctica federada entre 1 y 5 años (M: 3.04; DT: 1.10).

Tabla 1. Distribución de la muestra en función de la experiencia.

\begin{tabular}{lccc}
\hline Experiencia (años federados) & $\mathrm{N}$ & $\mathrm{M}(\mathrm{CP})$ & $\mathrm{DT}$ \\
\hline Alta experiencia (+ 5 años) & 38 & 16.15 & 3.32 \\
Experiencia Media (3 y 4 años) & 180 & 13.69 & 3.79 \\
Baja Experiencia (1 y 2 años) & 88 & 12.25 & 3.37 \\
Total & 306 & 13.31 & 3.77 \\
\hline
\end{tabular}

Tabla 2. Distribución de la muestra en función del rendimiento en clasificación.

\begin{tabular}{|c|c|c|c|}
\hline Rendimiento & $\mathrm{N}$ & $\mathrm{M}(\mathrm{CP})$ & DT \\
\hline $\begin{array}{l}\text { Alto rendimiento } \\
\text { (primeros clasificados, } 1^{\mathrm{o}}-4^{\mathrm{o}} \text { ) }\end{array}$ & 94 & 14.85 & 3.33 \\
\hline $\begin{array}{l}\text { Medio rendimiento } \\
\text { (posiciones intermedias, } 5^{\circ}-10^{\circ} \text { ) }\end{array}$ & 124 & 13.49 & 3.37 \\
\hline $\begin{array}{l}\text { Bajo rendimiento } \\
\text { (últimos clasificados, } 11^{\mathrm{o}}-18^{\circ} \text { ) }\end{array}$ & 88 & 11.64 & 4.01 \\
\hline Total & 306 & 13.31 & 3.77 \\
\hline
\end{tabular}

\section{Medida}

La variable dependiente del estudio fue el conocimiento procedimental:

- Conocimiento procedimental, identificado con el "saber cómo”. Es el conocimiento sobre cómo actuar, responde a una descripción sobre cómo hacer algo e incluye el procedimiento de selección apropiada de la respuesta dentro del contexto de juego (McPherson \& French, 1991). Para su medida se empleó la adaptación al voleibol elaborada por Moreno et al., (2013) a partir del cuestionario original de McGee \& Farrow, (1987). Está compuesto por un total de 25 preguntas con respuesta múltiple, de las cuales solo existe una correcta. Las preguntas planteadas en el cuestionario están relacionadas con el apartado de táctica del cuestionario original y reflejan cuestiones sobre diferentes situaciones tácticas o fases del juego existentes en voleibol: saque, recepción, colocación, ataque y defensa. Los jugadores deben resolverlo, seleccionando la opción o solución más apropiada para cada caso. El valor de fiabilidad del instrumento para la muestra de estudio fue de .79, considerado como aceptable por la literatura (Nunnally, Bernstein, \& Berge, 1967)

Las variables independientes consideradas en el estudio fueron las siguientes:

- Experiencia en práctica federada, entendida como los años de práctica en voleibol con licencia federativa. Se conformaron tres grupos diferentes: grupo de experiencia alta (5 ańos o más), un grupo de experiencia media (3 y 4 años de experiencia) y un grupo de experiencia baja (1 y 2 años de experiencia) (Ver tabla 1). Para su medida se atendió al historial de práctica de cada sujeto.

- Rendimiento, entendido como la posición obtenida en la clasificación por cada uno de los equipos al final del Campeonato. Se conformaron tres grupos diferentes, de tal manera que el grupo de rendimiento alto lo componían los $4^{\circ}$ primeros clasificados, el grupo de rendimiento medio quedó conformado por los clasificados del $5^{\circ}$ al $10^{\circ}$ y el grupo de rendimiento bajo lo componían los clasificados del $11^{\circ}$ en adelante (del $11^{\circ}$ al $18^{\circ}$ en femenino y del $11^{\circ}$ al $14^{\circ}$ en masculino) (Ver tabla 2). Para su medida se empleó la clasificación final de los equipos suministrada por la Real Federación Española de Voleibol.

\section{Procedimiento}

La toma de datos tuvo lugar durante el Campeonato de España de Voleibol de Selecciones Autonómicas de categoría infantil celebrado en Cartagena, Murcia (Espańa). El investigador principal fue el encargado de proporcionar los cuestionarios a los participantes, informar de cómo cumplimentar los mismos y solucionar posibles dudas que pudieran aparecer durante el proceso.

La investigación se realizó siguiendo las normas del Comité Ético de Investigación de la Universidad de Extremadura (2010), para la participación de los jugadores en el estudio y para la toma de datos. Tanto los entrenadores como los padres de los participantes dieron su consentimiento para poder acceder a la muestra, dando su autorización firmada.

\section{Análisis de datos}

De forma previa a la realización de los distintos análisis estadísticos, se practicaron pruebas de normalidad. Las medidas de asimetría, Curtosis y Kolmogorov-Smirnov con la corrección de Lilliefors verificaron que la distribución de la muestra era normal, por lo que se optó por el uso de la estadística paramétrica. En primer lugar, se realizó un análisis de varianza en función de la experiencia en práctica federada y el rendi- 
miento de los equipos, con el propósito de comprobar cómo ambas variables influyen en el conocimiento procedimental. El post-hoc de Tukey sirvió para determinar entre qué niveles de experiencia y rendimiento de los equipos existían diferencias significativas en la variable dependiente. El estadístico tamaño del efecto se tomó como referente para garantizar la adecuación de los resultados al instrumento y a la muestra de estudio, posibilitando su inclusión una mayor generalización de los mismos. Posteriormente, el análisis correlacional entre las variables experiencia en práctica federada, conocimiento procedimental y rendimiento en clasificación se empleó para comprobar el grado de asociación que existía entre dichas variables. El programa estadístico SPSS 19.0 sirvió de apoyo informático para el análisis de los datos recogidos

\section{Resultados}

Los resultados del análisis inferencial determinaron la existencia de diferencias en el conocimiento procedimental, en función de la experiencia en práctica federada $(\mathrm{F}(2,285)=10.685$; $\mathrm{p}<.001$; $\left.h p^{2}=.989\right)$ y el rendimiento $\left(\mathrm{F}(2,303)=44.434 ; \mathrm{p}<.001 ; \mathrm{hp}^{2}=1.00\right)$. El post-hoc de Tuckey permitió determinar la existencia de diferencias significativas en el conocimiento procedimental entre todos los niveles de la variable experiencia (Tabla 3 ) y entre todos los niveles de rendimiento (Tabla 4). Los elevados valores del tamaño del efecto corroboran el valor estadístico de las pruebas realizadas, así como la fiabilidad de los resultados obtenidos en base al instrumento y a la muestra de estudio.

Tabla 3. Post-Hoc de Tukey para el CP para la variable independiente experiencia

\begin{tabular}{llccc}
\hline Experiencia & & Dif. de medias & Error típico & Sig. \\
\hline \multirow{2}{*}{ Alta experiencia } & Experiencia media & $2.45^{*}$ & .85 & .013 \\
& Experiencia baja & $3.90^{*}$ & .90 & $<.001$ \\
Experiencia media & Experiencia alta & $-2.45^{*}$ & .85 & .013 \\
& Experiencia baja & $1.44^{*}$ & .47 & .007 \\
Baja experiencia & Experiencia alta & $-3.90^{*}$ & .90 & $<.001$ \\
\hline
\end{tabular}

*. La diferencia de medias es significativa al nivel .05

Tabla 4. Post-Hoc de Tukey para el CP para la variable independiente rendimiento

\begin{tabular}{llccc}
\hline Rendimiento & & Dif. de medias & Error típico & Sig. \\
\hline \multirow{2}{*}{ Alto rendimiento } & Rendimiento medio & 1.36 & .49 & .020 \\
& Rendimiento Bajo & $3.26^{*}$ & .53 & $<.001$ \\
\multirow{3}{*}{ Medio rendimiento } & Rendimiento alto & -1.36 & .49 & .020 \\
& Rendimiento bajo & $1.84^{*}$ & .48 & .001 \\
Bajo rendimiento & Rendimiento alto & $-3.20^{*}$ & .53 & $<.001$ \\
& Rendimiento medio & $-1.84^{*}$ & .48 & .001 \\
\hline
\end{tabular}

*. La diferencia de medias es significativa al nivel .05

Posteriormente, el análisis correlacional determinó asociación significativa entre las variables estudiadas (Tabla 5). Los resultados muestran pesos de asociación bajos aunque significativos entre las variables experiencia y rendimiento. Por otro lado el mayor peso de correlación se da entre el conocimiento procedimental y el rendimiento.

Tabla 5. Correlación de Pearson para las variables de estudio

\begin{tabular}{lcc}
\hline Variables & 1 & 2 \\
\hline 1. Conocimiento procedimental & - & \\
2. Experiencia en práctica federada & $.270^{* *}$ & \\
3. Rendimiento & $-.329^{* *}$ & $-.122^{*}$ \\
\hline$* *$ La correlación es significativa al nivel 0,01 (bilateral).
\end{tabular}

\section{Discusión}

El propósito fundamental de este estudio fue analizar el conocimiento de jugadores de voleibol en categoría infantil, en función de su experiencia en práctica federada y su rendimiento en clasificación.

La primera hipótesis de estudio determinaba que "existirán diferencias en el conocimiento procedimental de los jugadores en función de la experiencia en práctica federada, mostrando un conocimiento procedimental superior los jugadores con mayor experiencia en práctica federada”. Los resultados hallados permitieron confirmar esta primera hipótesis de estudio, al encontrarse diferencias significativas en el conocimiento procedimental en función de la experiencia 
en práctica federada. Específicamente, existieron diferencias entre jugadores de alta ( 5 años o más), media ( 3 y 4 años) y baja experiencia en práctica federada ( 1 y 2 años), siendo los jugadores con mayor experiencia los que mayor conocimiento procedimental manifestaron.

Estos resultados van en línea con trabajos previos que han tenido como propósito analizar cómo la variable experiencia en práctica influye en el desarrollo del conocimiento (Gil et al., 2013; Iglesias, García-González, García-Calvo, León, \& Del Villar, 2010; MacMahon \& Starkes, 2008 Del Villar, Iglesias, Moreno, Fuentes, \& Cervelló, 2004; Macquet, 2009; McPherson \& MacMahon, 2008).

Los resultados hallados son similares a los encontrados por Fernández-Echeverría et al., (2014) en un Campeonato de España de categoría cadete, reafirmándose las diferencias encontadas en conocimiento procedimental en los tres grupos de experiencia, siendo los más experimentados los que mostraron un conocimiento procedimental superior que los menos experimentados. De forma previa, Gil, Del Villar, Moreno, García-González, \& Moreno, (2011) en un trabajo en el que incluyeron las categorías de juego infantil, cadete y juvenil en voleibol, encontraron diferencias significativas en el conocimiento procedimental entre las diferentes categorías. Igualmente, en baloncesto de categoría infantil, como la del presente estudio. Del Villar et al., (2004) determinaron diferencias en el conocimiento procedimental de jugadores de alta experiencia (con una media de 3.87 años en competición), baja experiencia (con una media de 1.55 años en competición) y sin experiencia ( 0.49 años en práctica de baloncesto en las clases de educación física). Similares resultados, también en baloncesto, fueron hallados por French \& Thomas (1987) en un trabajo en el que los jugadores con mayor experiencia (infantiles) mostraron mayores valores de conocimiento que los menos experimentados (alevines). Podemos afirmar, en base a los resultados hallados y a los estudios previos, que los jugadores con más experiencia, independientemente de su edad, muestran mayores niveles de conocimiento que aquellos con menor experiencia (McPherson \&Thomas, 1989).

Se confirma de esta forma la idea de que la experiencia vivida por el deportista es muy relevante para el desarrollo del conocimiento en el deporte. Podemos destacar, de este modo, la importancia que tiene las experiencias previas vividas en diferentes situaciones y contextos deportivos, que podría enriquecer la base de conocimiento que los deportistas tienen sobre su propio deporte (Thomas et al., 1988).

La segunda hipótesis planteaba que "existirán diferencias en el conocimiento procedimental de los jugadores en función del rendimiento en clasificación, mostrando un conocimiento procedimental superior los jugadores pertenecientes a equipos mejor clasificados". Los resultados hallados permitieron confirmar esta segunda hipótesis de estudio. Se encontraron diferencias significativas en el conocimiento procedi- mental en función del rendimiento en clasificación, siendo el conocimiento procedimental de los jugadores pertenecientes a los equipos mejor clasificados significativamente superior al de los jugadores clasificados en posiciones intermedias y finales de la tabla.

Estos resultados van en línea con otros estudios, también en categorías de formación, que determinaron la existencia de diferencias en el nivel de conocimiento en función del rendimiento obtenido por los equipos en clasificación (FernándezEcheverría et al., 2014). Anteriormente, en un estudio con jugadores de baloncesto en categoría infantil, Del Villar et al., (2004) encontraron que aquellos equipos cuyos jugadores poseían un mayor nivel de conocimiento procedimental, ocuparon las posiciones más altas en la tabla clasificatoria. En 2006, Moreno, Moreno, García-González, et al. establecieron una relación lineal entre el conocimiento y el rendimiento, de tal manera que a mayor conocimiento, mayor rendimiento en clasificación. En un estudio previo en baloncesto, French \& Thomas (1987), compararon dos categorías diferentes de juego, hallando que los jugadores con mayor nivel de conocimiento sobre el baloncesto también alcanzaron mejor rendimiento, en este caso en la ejecución del tiro a canasta. Podemos interpretar que esa base de conocimiento procedimental podría mejorar capacidad de la selección de respuesta más adecuada y contextualizada a la situación de juego, lo que podría hacer que los jugadores alcanzaran mayores cotas de rendimiento individual y posibilitando la mejor actuación colectiva en clasificación.

La tercera hipótesis de estudio planteaba que "existirá asociación significativa entre el conocimiento procedimental, la experiencia en práctica federada y rendimiento en clasificación de los jugadores". Los resultados hallados en el presente estudio permiten confirmar la tercera hipótesis de estudio. Se halló asociación significativa del conocimiento procedimental con el rendimiento y con la experiencia, aunque los reducidos pesos de correlación hacen que estos datos deban ser interpretados con cautela.

Los resultados obtenidos coinciden con investigaciones previas en diferentes deportes que han llegado a relacionar la experiencia con el rendimiento en juego (McMorris \& Beazeley, 1997; Williams, Davids, Burtwitz, \& Williams, 1993) o en clasificación (Moreno, Moreno, García-González, et al., 2006); la experiencia con el conocimiento (Bläsing, Tenenbaum, \& Schack, 2009; García-González, Iglesias, Moreno, Gil, \& Del Villar, 2011; Mesquita, Graça, Gomes, \& Cruz, 2005); y el conocimiento con el rendimiento tanto en juego como en clasificación (García-González et al., 2011; Gil, Moreno, et al., 2011b; Moreno, Moreno, García-González, et al., 2006).

Estos resultados concuerdan con otros trabajos en voleibol en categorías de formación (Del Villar et al., 2004; Moreno, Moreno, García-González, et al., 2006), que encontraron 
asociación entre el conocimiento y el rendimiento en clasificación. Sin embargo, no concuerdan con los hallados por (Fernández-Echeverría et al., 2014) en categoría cadete en cuanto a la asociación del conocimiento procedimental y el rendimiento.

También categorías de formación Del Villar et al., (2004) en categoría infantil de baloncesto o Moreno, Moreno, García-González, et al., (2006) en voleibol, analizaron la relación existente entre conocimiento procedimental, experiencia y rendimiento en jóvenes jugadores con diferentes niveles de pericia. En ambos deportes se halló una relación lineal y significativa entre las variables experiencia en federado y rendimiento, y entre conocimiento procedimental y rendimiento.

Las variables conocimiento procedimental, experiencia y rendimiento han sido y siguen siendo objeto de multitud de investigaciones, lo que pone de manifiesto su relevancia en el proceso que lleva a los jugadores hacia la excelencia deportiva. En este sentido, este trabajo profundiza en el análisis del conocimiento procedimental de los jugadores del Campeonato de España de categoría infantil, en función de la experiencia federada y el rendimiento de sus equipos en clasificación. Se considera necesario que los entrenadores desarrollen situaciones de entrenamiento para la adquisición de este conocimiento procedimental, con los objetivos de mejorar la resolución de problemas tácticos en etapas formativas (Dodds, Griffin, \& Placek, 2001) y de optimizar el proceso de información deportiva iniciación deportiva

\section{Referencias}

1. Abernethy, B., Farrow, D., \& Berry, J. (2003). Constraints and issues in the development of a general theory of expert perceptual-motor performance: a critique of the Deliberate Practice framework. In J. L. Starkes (Ed.), Expert performance in sport: recent advances in research on sport expertise (pp. 349-369). Champaign, Illinois: Human Kinetics.

2. Abernethy, B., Thomas, K., \& Thomas, J. (1993). Strategies for improving understanding of motor expertise (or mistakes we have made and things we have learned!!). In J. Starkes \& F. Allard (Eds.), Cognitive issues in motor expertise (p. 317). Amsterdam: Elsiever.

3. Alexander, P., \& Judy, J. (1988). The interaction of domain-specific and strategic knowledge in academic performance. Review of Educational Research, 58(4), 375-404.

4. Anderson, A., Knowles, Z., \& Gilbourne, D. (2004). Reflective practice for sport psychologists: concepts, models, practical implications, and thoughts on dissemination. The Sport Psychologist, 18, 188-203.

5. Anderson, J. (1987). Skill acquisition: Compilation of weak-method problem situations. Psychological Review, 94(2), 192-210.

6. Araújo, D. (2005). O contexto da decisao. A acçao táctica no desporto. Lisboa: Visao e Contextos.

7. Araújo, D. (2006). Tomada de decisao no desporto. Cruz Quebrada, Portugal: Ediçoes FHM.

8. Araujo, D., \& Davids, K. (2009). Ecological approaches to cognition and action in sport and exercise: Ask not only what you do, but where you do it. International Journal of Sport Psychology, 40(1), 5-37.

9. Araújo, D., Davids, K., Cordovil, R., Ribeiro, J. y Fernandes, O. (2009). How does knowledge constrain sport perfomance? An ecological perspective. En D. Araújo, H. Ripoll y M. Raab (Eds.) Perspectives on Cognition and Action in Sport (pp. 119-131). New York:Nova Science Publishers.

10. Araujo, D., Davids, K., y Hristovski, R. (2006). The ecological dynamics of decision making in sport. Psychology of Sport and Exercise, 7 , 653-676.

11. Bläsing, B., Tenenbaum, G., \& Schack, T. (2009). The cognitive structure of movements in classical dance. Psychology of Sport and Exercise, 10(3), 350-360. doi:10.1016/j.psychsport.2008.10.001

12. Browne, T., Carlson, T., \& Hastie, P. (2004). A Comparison of Rugby Seasons Presented in Traditional and Sport Education Formats. European Physical Education Review, 10(2), 199-214. doi:10.1177/1356336X04044071

13. Chi, M. (1978). Knowledge structures and memory development. In R. Siegler (Ed.), Children's thinking: What develops (pp. 73-96). Hillsdale, NJ: Erlbaum.
14. Del Villar, F., Iglesias, D., Moreno, M. P., Fuentes, J. P., \& Cervelló, E. (2004). An investigation into procedural knowledge and decisión-making: spanish experienced- inexperienced basketball players differences. Journal of Human Movement Studies, 46, 407-420.

15. Dodds, P., Griffin, L., \& Placek, J. (2001). A selected review of the literature on development of learners' domain-specific knowledge. Journal of Teaching in Physical Education, 20, 301-313.

16. Fernández-Echeverría, C., Moreno, A., Gil, A., Claver, F., \& Moreno M. P. (2014). Estudio del conocimiento procedimental, experiencia y rendimiento, en jóvenes jugadores de voleibol. Retos. Nuevas Tendencias En Educación Física, Deporte Y Recreación, 25(1), 13-16.

17. French, K., \& Thomas, J. (1987). The relation of knowledge development to children's basketball performance. Journal of Sport Psychology, 9(1), 15-33.

18. García-López, L., González-Víllora, S., Gutiérrez, D., \& Serra, J (2013). Desarrollo y validación de la herramienta de evaluación del rendimiento de juego (HERJ) en fútbol. Sportk. Revista Euroamericana de Ciencias Del Deporte, 1(2), 89-99.

19. García-González, L., Iglesias, D., Moreno, A., Gil, A., \& Del Villar F. (2011). La competición como variable precursora del conocimiento en tenis. Revista Internacional de Medicina Y Ciencias de La Actividad Fisica Y Del Deporte, 11(43), 1-16.

20. Gil, A., Del Villar, F., Moreno, A., García-González, L., \& Moreno, M P. (2011). Análisis de la eficacia del saque de voleibol en categoría de formación. Revista Internacional de Medicina Y Ciencias de La Actividad Física Y El Deporte, 44(11), 721-737.

21. Gil, A., Moreno, M. P., Moreno, A., García-González, L., Claver, F., \& Del Villar, F. (2013) Analysis of the relationship between the amount of practice in training and cognitive expertise: a study on young volleyball players. Journal of Strength and Conditioning Research, 27(3)/698-702

22. Gil, A., Moreno, M. P., Moreno, A., García-González, L., \& Del Villar, F. (2011a). Estudio del saque en jóvenes jugadores/as de voleibol, considerando la eficacia y función en juego. Retos. Nuevas Tendencias En Educación Física, Deporte Y Recreación, 19, 19-24.

23. Gil, A., Moreno, M. P., Moreno, A., García-González, L., \& Del Villar, F. (2011b). La práctica federada como elemento de desarrollo del conocimiento: aplicación al voleibol de formación.. RICYDE. Revista Internacional de Ciencias Del Deporte, 7(24), 230-245. doi:10.5232/ricyde2011.02406

24. Iglesias, D., García-González, L., García-Calvo, T., León, B., \& Del Villar, F. (2010). Expertise development in sport: contributions under cognitive psychology perspective. Journal of Human Sport \& Exercise, 5(3), 462-475. doi:10.4100/jhse.2010.53.16 
25. MacMahon, C., \& McPherson, S. (2009). Knowledge base as a mechanism for perceptual-cognitive tasks ... Knowledge base as a mechanism for perceptual-cognitive tasks : skill is in the details. International Journal of Sport Psychology, 40(4), 565-579.

26. MacMahon, C., \& Starkes, J. L. (2008). Contextual influences on baseball ball-strike decisions in umpires, players, and controls. Journal of Sports Sciences, 26(7), 751-60. doi:10.1080/02640410701813050

27. Macquet, A. (2009). Recognition Within the Decision-Making Process: A Case Study of Expert Volleyball Players. Journal of Applied Sport Psychology, 21(1), 64-79. doi:10.1080/10413200802575759

28. McGee, R., \& Farrow, A. (1987). Test questions for physical education activities. Test questions for physical education activities (pp. 23-25). Champaign, IL: Human Kinetics.

29. McMorris, T., \& Beazeley, A. (1997). Performance of experienced and inexperienced soccer players on soccer specific tests of recall, visual search and decision-making. Journal of Human Movement Studies, 33, $1-13$.

30. McPherson, S. (1994). The Development of Sport Expertise: Mapping the Tactical Domain. Quest, 46(2), 223-240. doi:10.1080/00336297.1 994.10484123

31. McPherson, S. (1999). Expert-Novice Differences in Performance Skills and Problem Representations of Youth and Adults during Tennis Competition. Research Quarterly for Exercise and Sport, 70(3), 233-251. doi: 10.1080/02701367.1999.10608043

32. McPherson, S., \& French, K. (1991). Changes in cognitive strategies and motor skill in tennis. Journal of Sport \& Exercise, 13(1), 26-41.

33. McPherson, S. L., \& Thomas, J. R. (1989). Relation of knowledge and performance in boys' tennis: age and expertise. Journal of Experimental Child Psychology, 48(2), 190-211.

34. McPherson, S., \& MacMahon, C. (2008). How baseball players prepare to bat: Tactical knowledge as a mediator of expert performance in baseball. Journal of Sport \& Exercise Psychology, 30(6), 755-78.

35. Mesquita, I., Graça, A., Gomes, R., \& Cruz, C. (2005). Examining the impact of a step game approach to teaching volleyball on student tactical decision making and skill execution during game play. Journal of Human Movement Studies, 48, 1-28.

36. Moreno, A., Del Villar, F., García-González, L., García-Calvo, T., \& Moreno, M. P. (2013). Propiedades psicométricas de un cuestionario para la evaluación del conocimiento procedimental en voleibol (CCPV).
RICYDE. Revista Internacional de Ciencias Del Deporte, 30(9), 38-47. doi:10.5232/ricyde2011.02602

37. Moreno, A., Moreno, M. P., García-González, L., García-Calvo, T., \& Del Villar, F. (2010). Diferencias en la planificación de estrategias en voleibol entre jugadoras expertas y noveles. Apunts Educació Física $Y$ Deportes, 102(4), 31-37.

38. Moreno, A., Moreno, M. P., García-González, L., Iglesias, D., \& Del Villar, F. (2006). Relación entre conocimiento procedimental, experiencia y rendimiento. Un estudio en voleibol. Motricidad. European Journal of Human Movement, 17, 15-24.

39. Moreno, A., Moreno, P., Iglesias, D., García-González, L., \& Del Villar, F. (2006). Estudio del conocimiento declarativo en función de la experiencia y de la edad en jugadores jóvenes de voleibol. Cultura, Ciencia $Y$ Deporte, 5(2), 73-80.

40. Newell, K. (1996). Change in movement and skill: Learning, retention, and transfer. In M. Latash \& M. Turvey (Eds.), Dexterity and its development (pp. 393-429). Mahwah, NJ: Erl.

41. Nunnally, J., Bernstein, I., \& Berge, J. (1967). Psychometric theory Nueva York: McGraw Hill

42. Tenenbaum, G., Yuval, R., Elbaz, G., Bar-Eli, M., \& Weinberg, R. (1993). The relationship between cognitive characteristics and decision making. Canadian Journal of Applied Physiology, 18(1), 48-62.

43. Thomas, J., French, K., Thomas, K., \& Gallagher, J. (1988). Children's knowledge development and sport performance. In I. Srnod, R.Magill, \& J. Ash (Eds.), Children in sport (pp. 179-202). Champaign, IL: Human Kinetics.

44. Thomas, K. (1994). The Development of Sport Expertise: From Leeds to MVP Legend. Quest, 46(2), 199-210. doi:10.1080/00336297.1994.1 0484121

45. Thomas, K., \& Thomas, J. (1994). Developing expertise in sport: The relation of knowledge and performance. International Journal of Sport Psychology, 25, 295-312.

46. Williams, A. M., \& Ericsson, K. A. (2005). Perceptual-cognitive expertise in sport: some considerations when applying the expert performance approach. Human Movement Science, 24(3), 283-307. doi:10.1016/j. humov.2005.06.002

47. Williams, A.M., Davids, K., Burtwitz, L., \& Williams, J. (1993). Cognitive knowledge and soccer performance. Perceptual and Motor Skills, $76,579-593$. 